\title{
Improvement of Magnetic Exploration Device Using Differential Magnetic Sensor for Detecting Unexploded Ordnance
}

\author{
Hiroshi Kawano, $^{\mathrm{a},{ }^{*}, \text { Seiichi Serikawa }}{ }^{\mathrm{b}}$ \\ ${ }^{a}$ MTI Corporation, 2-1-15 Edamitsu, Yahata-higashi, Kitakyushu, Fukuoka, 805-8503, Japan \\ ${ }^{\mathrm{b}}$ Kyushu Institute of Technology, 1-1 Sensui, Tobata, Kitakyushu, Fukuoka, 804-8550, Japan \\ *Corresponding Author: kawano@ mticorp.co.jp
}

\begin{abstract}
A new method to detect unexploded ordnance is proposed in this study. Magnetic exploration system using a plurality of differential magnetic sensor with different distance is used. By comparing the plurality of differential outputs simultaneously, we can reduce the influence of the magnetic field from outside of the search-area. Unexploded ordnance will be detected correctly in spite of the place where conventional method cannot detect.
\end{abstract}

Keywords: unexploded ordnance, Magnetic exploration, magnetic detecting device.

\section{Introduction}

During World War II, in Japan many bombs were dropped by large-scale air strikes. Sometimes, the bombs did not explode. Such bomb is called unexploded ordnance. In Okinawa, there happened the intense ground war. Unexploded ordnance which was brought by aircraft bombs and naval gunfire still exists now. For this reason, the exploration of unexploded ordnance is often carried out to keep safety in Okinawa ${ }^{(1)}$.

The structure with a ferromagnetic material near search-area often causes the false detection of unexploded ordnance. This is the problem for the exploration. By the magnetic field from outside the search-area, the output of exploration device increases. Sometimes, the value is greater than the threshold. As the result, the device judges that there exists unexploded ordnance, in spite that it does not existent. That is wrong judgment.

In this paper, we propose a new method to solve this problem. Magnetic exploration system using a plurality of differential magnetic sensor with different distance is used.
By comparing the plurality of differential outputs simultaneously, we can reduce the influence of the magnetic field from outside of the search-area.

\section{Conventional Research}

\subsection{Method for Exploration}

As a method for exploration of unexploded shells, there is a method of electromagnetic induction method ${ }^{(2)}$ and a method of differential magnetic detection ${ }^{(3)}$. A metal detector is well known as the representative of electromagnetic induction method. This is well used in airport baggage inspection and detection of landmine. The device detects an eddy current by electromagnetic induction. It has directional sensitivity, so does not get the influence of the magnetic field from the adjacent area. However, the detecting range of distance is small compared with the method of differential magnetic detection. That is at most several $10[\mathrm{~cm}]$. Therefore, this method can be used to detect mines that are placed near the surface. It is not suitable for detection of aerial bombs, because it is buried under underground several meters.

The method of differential magnetic detection gets the difference between the two magnetic sensor outputs. These sensors are attached to both ends of the rod-like probe. The device using this method is called magnetic detecting device. The detection range of the device is 4-6 [m]. However, this is not useful for detecting non-ferrous metals, because of the use of magnetic sensor.

\subsection{Magnetic Detecting Device}

The characteristic of magnetic detecting device depends on the type of magnetic sensor. The search coil 
type of device is widely used, because magnetic sensor probe can be easily made at low cost. It is the device that detects the difference between both magnetic fluxes passing through coil-ends while moving. However, when the sensor does not move, it can not detect a magnetic field. In addition, since the change of moving-speed affects the change of sensitivity, it causes an error if the moving speed does not keep constant. For this problem, we do not adopt search coil type device in this study.

The fluxgate type of magnetic detecting device is used in this study. This is explained here. It is complex and expensive compared to the search coil magnetic sensor-type device. However, it is possible to detect the magnetic field even when the sensor does not move. In addition, the output value of the sensor does not change even if the movement speed changes. In the device, there are two types. One type of device has three-axis sensor, and another has one-axis sensor. The device with three-axis sensor has an ability of advanced analysis. However, it is often complicated and expensive. In addition, the handling is difficult.

As the other devices, there are the device with optical pumping magnetic sensor ${ }^{(4)}$ and a nuclear magnetic resonance sensor ${ }^{(5)}$, et al. These devices are more sensitive and stable than the device with fluxgate type of magnetic sensor. However, these are very expensive, the sizes are large, and they have much complicated constructions. Therefore, they are used only in special applications such as submarine exploration.

Thus, this study adopts one-axis fluxgate type of magnetic detecting device.

\section{Proposed Method}

\subsection{Principle}

Conventional magnetic detecting device adopts the method using a single differential output. To get the differential output, two magnetic sensors are attached to both ends of the rod-like probe.

In this study, three magnetic sensors are attached. By comparing the change in the plurality of differential output, the influence of the magnetic field from the adjacent area will be reduced. This is our purpose. Figure 1 shows the layout of the device with fluxgate type of magnetic sensor used in this study. In this figure, each sensor is called S1, S2, and S3, respectively. Let the distance between S1 and $\mathrm{S} 2$ be D1, let the distance between $\mathrm{S} 2$ and $\mathrm{S} 3$ be D2, and let the distance between S1 and S3 be D3. The values of D1,

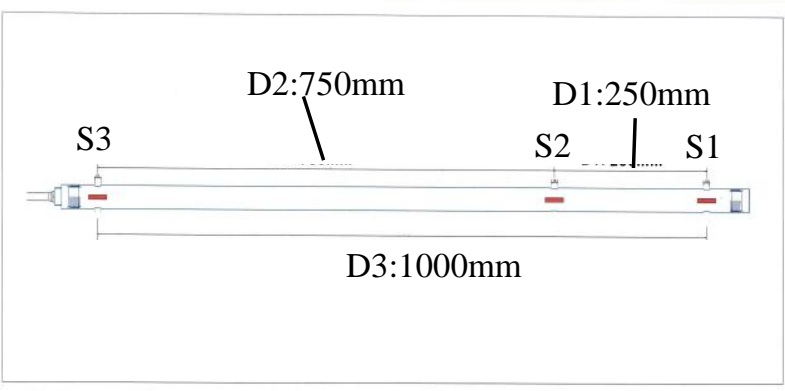

Fig. 1. Layout of fluxgate type of magnetic sensor.

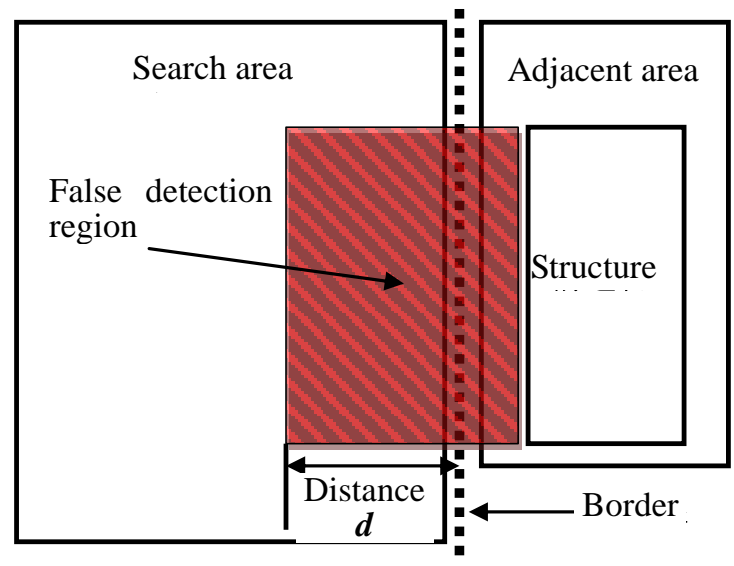

Fig. 2. Influence of the magnetic field from the adjacent area.

D2 and D3 are $250[\mathrm{~mm}], 750[\mathrm{~mm}]$ and $1000[\mathrm{~mm}]$, respectively.

\subsection{Influence of the Magnetic Field from the Adjacent Area}

Figure 2 shows the influence of the magnetic field from the adjacent area. The shaded area in Fig. 2 shows the area where false detection happens due to the influence of the magnetic field from the adjacent structures. In the figure, the distance $d$ is $4-6[\mathrm{~m}]$. Although there are many structures with ferromagnetic material, a rebar is considered in our study. This is because a rebar is well used in concrete buildings. As for rod-like material such as rebar, considering the macro area, the magnetic field distribution is approximated as the distribution of material with a linear dipole ${ }^{(6),(7)}$. In this case, the magnitude of the magnetic field is inversely proportional to the square of the distance ${ }^{(8)}$.

\subsection{Simulation of Magnetic Field Distribution}

Figure 3 shows the layout for the simulation of the distribution of the magnetic field caused by ferromagnetic material with a linear dipole. Here, a linear dipole is located at the position of $5[\mathrm{~m}]$ to $X$ axis. The length of linear dipole is $4[\mathrm{~m}]$, and it has two poles of $+2.0[\mathrm{mWb}]$ and 


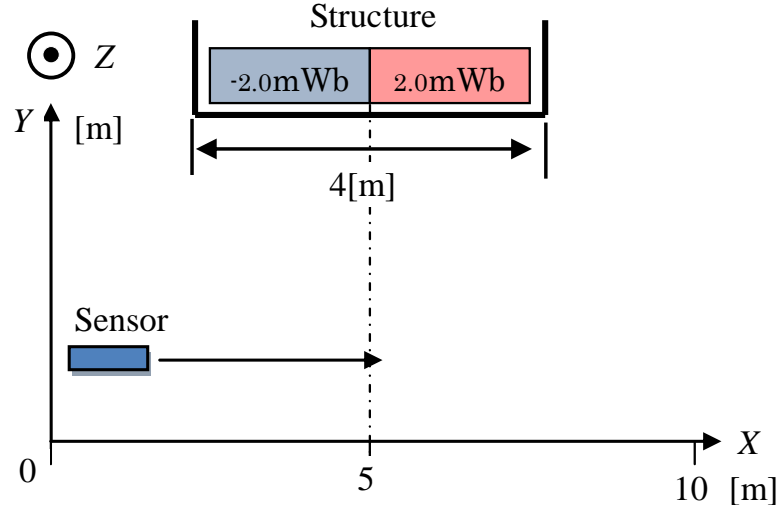

Fig. 3. Layout for the simulation of the distribution of the magnetic field caused by ferromagnetic material with a linear dipole.

$+2.0[\mathrm{mWb}$. This is a simulation of the rebar in the wall of the structure. While keeping distance 2, 4 and 6 [m] from the structure to $\mathrm{Y}$ axis, the single magnetic sensor moves 0-10 [m] to $X$ axis. Here, one-axis type of magnetic sensor is used. Then, the magnetic field distribution to $X$ axis is shown in Figs.4 (a), (b) and (c). In Fig. 4, the negative sign of output agrees with the negative direction of $\mathrm{X}$ axis, and the positive sign is the positive of $\mathrm{X}$ axis. In general, a rebar in wall is assembled in a lattice direction of $\mathrm{X}$ and $\mathrm{Z}$ axes. However, the magnetic output brought by rebar along the $\mathrm{Z}$ axis is sufficiently small compared to that along $\mathrm{X}$ axis. Thus, the rebar along $\mathrm{Z}$ axis may be ignored at this simulation. Next, the differential sensor probe in Fig. 1 moves in the space where the distribution in Fig. 4 exists. The result is shown in Fig. 5. It shows the relationship between the distance of $\mathrm{X}$ axis and the differential output of the sensor.

In Fig. 5, it has a negative peak at around $3[\mathrm{~m}]$ in $X$ axis. Table 1 shows the negative peak value of each graph in Fig. 5. Here, $1[\mathrm{mOe}]$ is regarded to $100[\mathrm{nT}]$ in this simulation.

\section{Experiment}

\subsection{Measurement of Magnetic Field Distribution}

We actually made the probe device shown in Fig. 1, and prepared the space similar to Fig.3. At the condition, the magnetic field distribution was measured. Here, the distance toward $\mathrm{Y}$ axis was set to $6[\mathrm{~m}]$ in the same manner as Fig. 5 (c). The device was moved 10 [m] along $\mathrm{X}$ axis. The measured distribution is shown in Fig. 6. In spite that the moving speed of the $\mathrm{X}$ direction is not actually constant, the output waveform is similar to that in Fig. 5 (c).

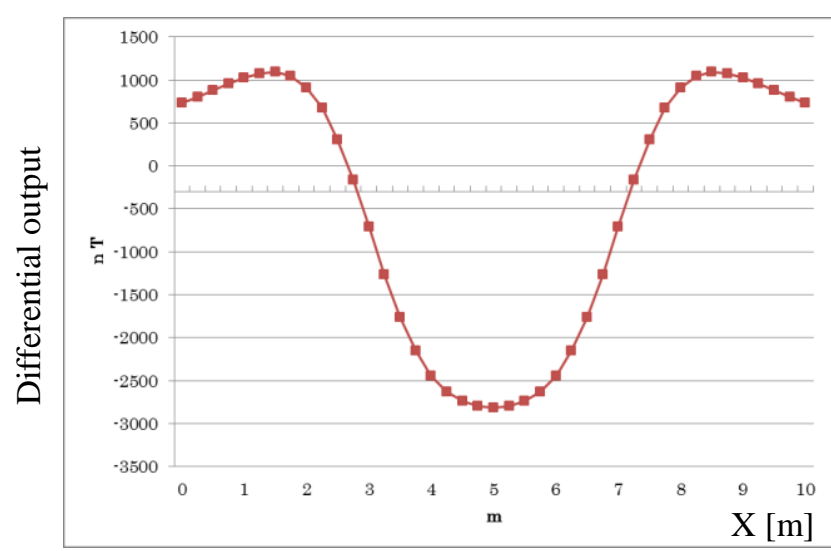

(a) $\mathrm{Y}=2[\mathrm{~m}]$

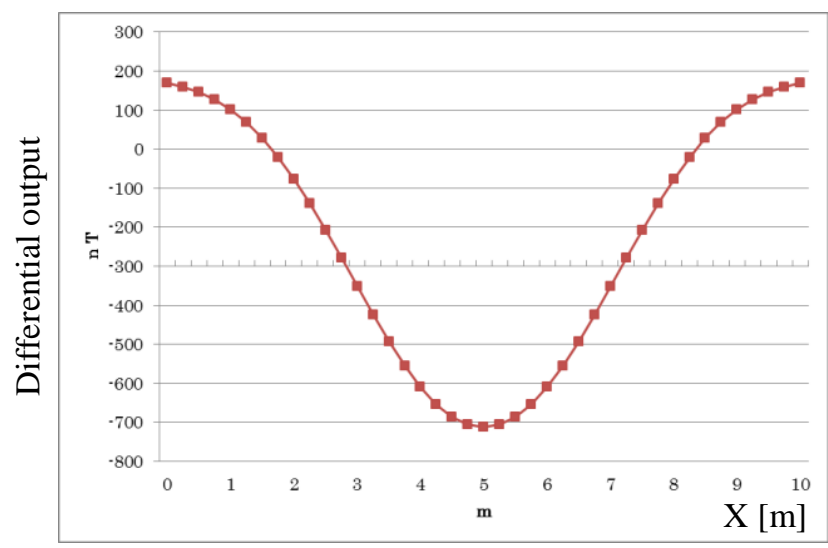

(b) $\mathrm{Y}=4[\mathrm{~m}]$

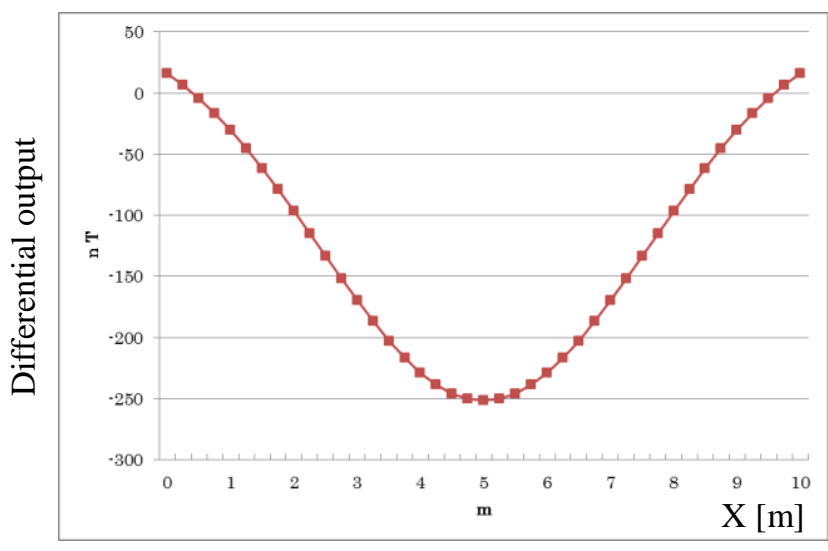

(c) $\mathrm{Y}=6[\mathrm{~m}]$

Fig. 4. Magnetic field distribution to $\mathrm{X}$ axis.

\subsection{Performance Verification}

We put a ferromagnetic material with the width 40 [cm], within the false detection region shown in Fig. 2. In this study, the material is called test object, and that is regarded to unexploded ordnance. While moving the sensor, the differential output waveform was measured. The test 


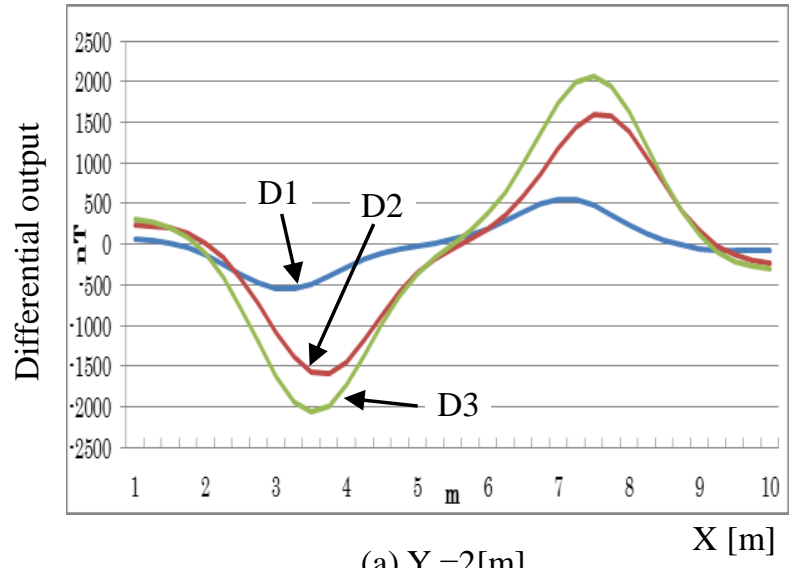

(a) $\mathrm{Y}=2[\mathrm{~m}]$

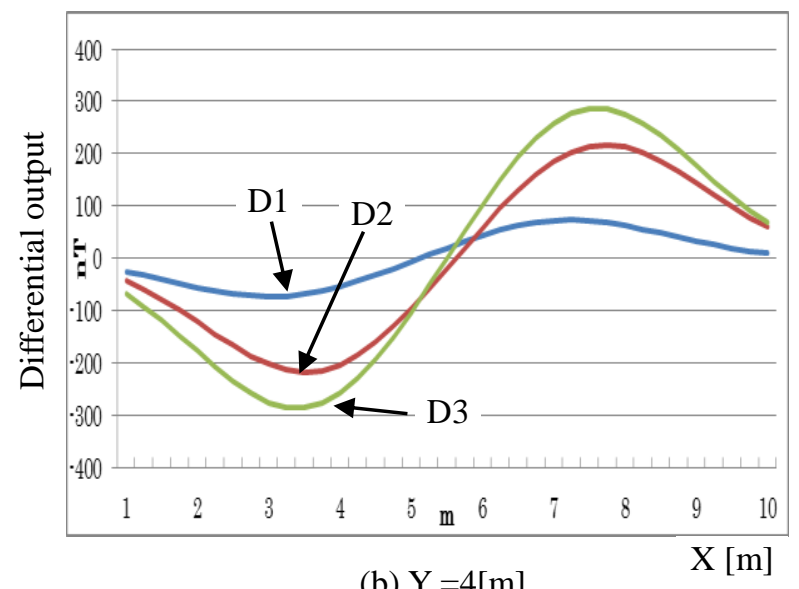

(b) $\mathrm{Y}=4[\mathrm{~m}]$

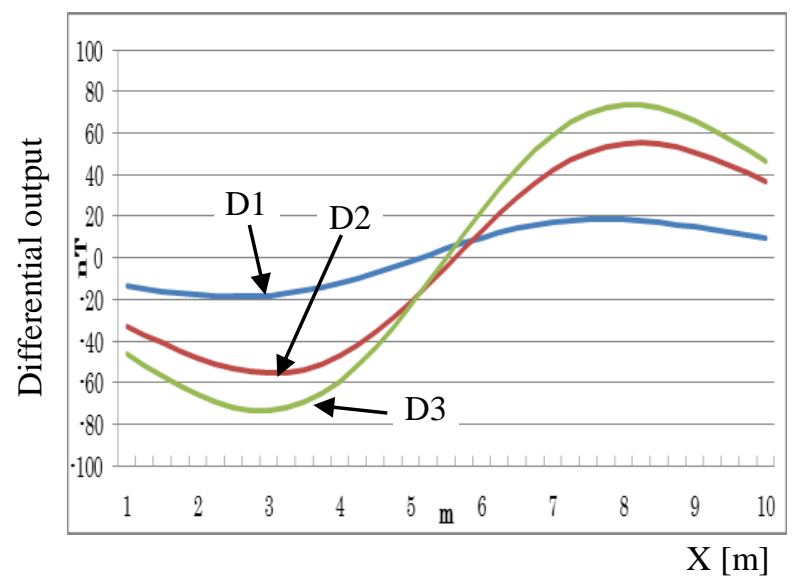

(c) $\mathrm{Y}=6[\mathrm{~m}]$

Fig. 5. Relationship between the distance in $\mathrm{X}$ direction and the differential output of the sensor.

Table 1. Negative peak value of each graph in Fig. 5.

\begin{tabular}{|c|c|c|c|}
\hline Y axis & D1 & D2 & D3 \\
\hline $2[\mathrm{~m}]$ & $-551 \mathrm{nT}$ & $-1591 \mathrm{nT}$ & $-2067 \mathrm{nT}$ \\
\hline $4[\mathrm{~m}]$ & $-73 \mathrm{nT}$ & $-217 \mathrm{nT}$ & $-285 \mathrm{nT}$ \\
\hline $6[\mathrm{~m}]$ & $-18 \mathrm{nT}$ & $-55 \mathrm{nT}$ & $-73 \mathrm{nT}$ \\
\hline
\end{tabular}

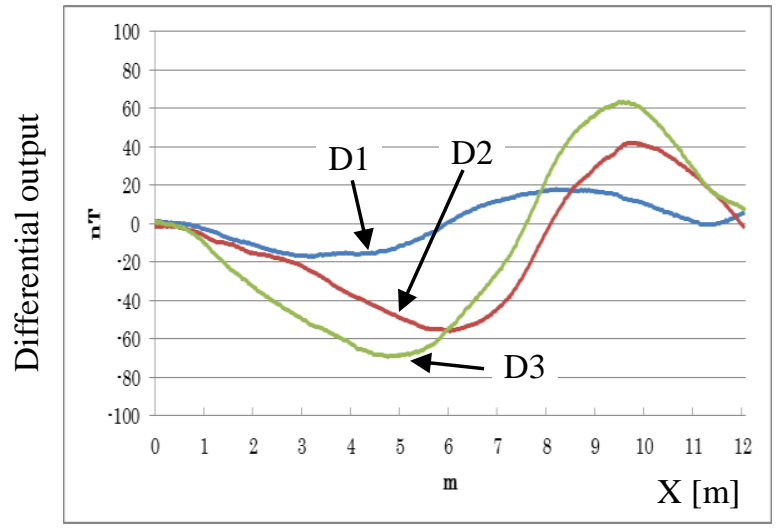

Fig. 6. Relationship between the distance in $\mathrm{X}$ direction and the differential output of the sensor.

(Y=6[m], Measured value)

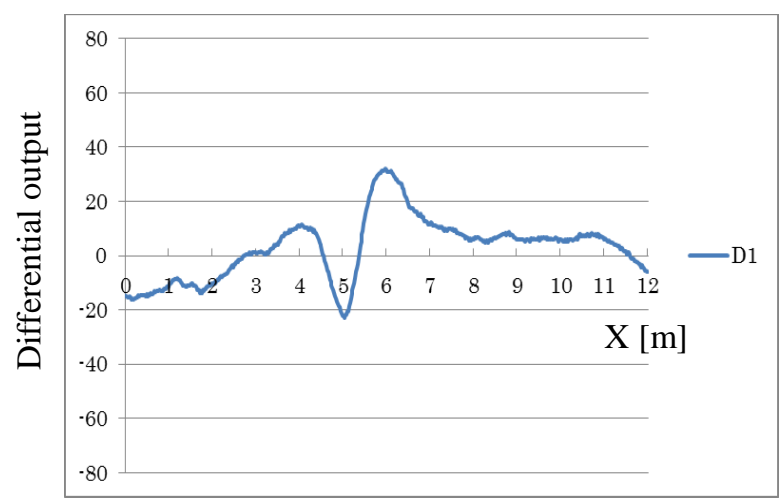

(a) Output of D1.

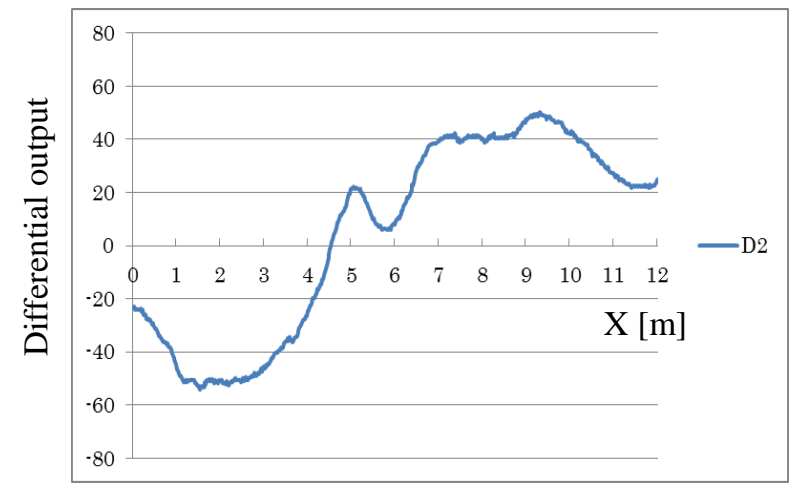

(b) Output of D2.

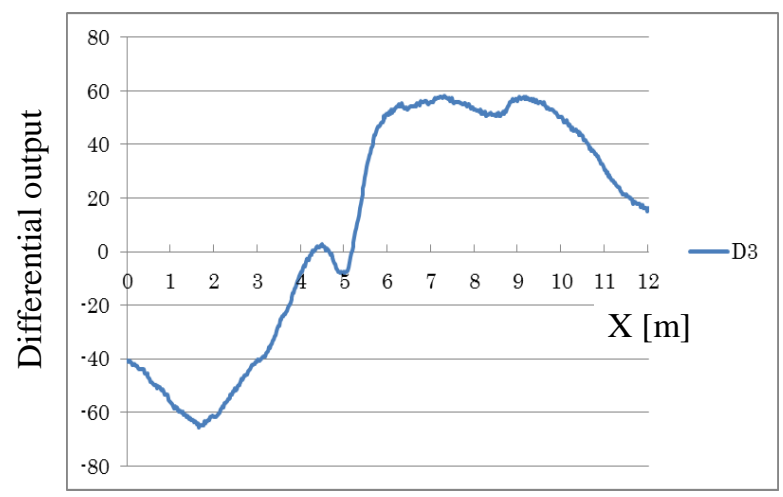

(c) Output of D3.

Fig. 7. Differential output when the device is moved in the direction of $\mathrm{X}$ axis. 
object was placed at the position of $5[\mathrm{~m}]$ in $\mathrm{X}$ axis and 4 [m] in $\mathrm{Y}$ axis. The sensor was moved $10[\mathrm{~m}]$ in the direction of $\mathrm{X}$ axis. Then, each differential output was measured. The results are shown in Figs. 7 (a)-(c). Figure 7 is roughly similar to Fig. 6. The large fluctuation ranging from negative peak to positive peak is confirmed in Fig. 7. The values of peak to peak are about 100 [nT] in D2, and 120 [nT] in D3. As understood from the Fig. 7 (a), the magnetic change at the position of around $5[\mathrm{~m}]$ can be easily found. Contrary to the figure, the change is unclear from the waveforms of D2 and D3 in Figs. 7 (b) and (c). Therefore, we can identify the test object. This means that test object such as unexploded ordnance can be detected in spite of false detection region.

In this test object, we can detect unexploded ordnance from the waveform D1. If the size of object changes, the waveform of D1 will also changes. If the size increases, the waveform of D2 and D3 will change to the waveform such as Fig. 7 (a). In general, when we use the sensor for unexploded ordnance, the size of unexploded ordnance is unclear yet. Thus, by simultaneously comparing differential outputs of D1, D2 and D3, we can identify the unexploded ordnance of various sizes.

\section{Conclusions}

A new method to detect unexploded ordnance is proposed in this study. Magnetic exploration system using a plurality of differential magnetic sensor with different distance is used. By comparing the plurality of differential outputs simultaneously, we can reduce the influence of the magnetic field from outside of the search-area. Thus, unexploded ordnance can be detected correctly in spite of the place where conventional method cannot detect.

\section{References}

(1) Okinawa General Bureau : "Measures such as unexploded ordnance in Okinawa", Shimatatei, No. 52, pp. 44-45, 2010

(2) N. I. HuthA and P. L. Poulton : "An electromagnetic induction method for monitoring variation in soil", Australian Journal of Soil Research, Vol. 45, pp. 63-72, 2007

(3) Lemarquand, Valerie and Annecy-le-Vieux : "Magnetic differential torque sensor", IEEE Transactions on Magnetics, Vol. 31, No. 6, pp. 3188-3190, 1995

(4) Avrin, William F. and Sager, Ronald E. : “An optically pumped magnetic sensor with submilligamma resolution", IEEE Transactions on Magnetics, Vol. 25, No. 5, pp. 3408-3410, 1989

(5) Goswami, Jaideva C., Sezginer, Apo. and Luong, Bruno : "On the design of NMR sensor for well-logging applications", IEEE Transactions on Antennas and Propagation, Vol. 48, No. 9, pp. 1393-1402, 2000

(6) Soushin Chikamizu : "Physics in Ferromagnetic", Syokabo", pp. 95-112, 1965, in Japanese

(7) Akira Mogi : "Introduction to magnetic application", Nikkangokyo-shinbunsha, pp. 95-112, 1972, in Japanese

(8) Jyunji Suyama : "Aeromagnetic exploration", Chishitsu News, No. 133, pp. 34-39, 1965, in Japanese 